\title{
Safe Navigation for Indoor Mobile Robots Part I: A Sensor-based Navigation Framework
}

\author{
Alessandro Corrêa Victorino, Patrick Rives, Jean-Jacques Borrelly
}

\begin{abstract}
This paper presents a first part, Part-I of two, of a complete exploration and navigation methodology that enables a robot to safely navigate in an unknown environment. Reactive sensor-based navigation tasks and control laws are derived from the interaction between the robot and its workspace. The perception is performed by a 2-D laser range finder mounted on the robot. Reactive navigation tasks are defined based on the task function framework [18] in a such a way that the robot can explore an unknown indoor environment without any reference trajectory computation. Obstacle avoidance is ensured as a straight property implicit to the definition of the navigation tasks. The stability and robustness of the derived control laws with respect to the model errors are analyzed. The experimental results validate the proposed methodology.
\end{abstract}

\section{INTRODUCTION}

The navigation of an autonomous mobile robot in an unknown environment requires some essential capabilities such as the perception of its environment and the capability of moving in a safe way, that is avoiding eventual obstacles. Some classes of solutions to this safe navigation problem are proposed in the literature distributed between path planning and motion control.

Classically, path planning methods address the existence and the generation of feasible collision-free trajectories between the initial and the goal positions in the robot workspace. A known map of the environment is required a priori [1], [11], [8]. The execution of such feasible collision-free trajectories considering the uncertainties concerns the motion control issues. In [10] the aspects of trajectory planning and motion control are treated in a common framework. A motion planner is presented in [7] where sensor-based actions are developed based on a task-potential field approach.

Feedback controllers have been designed in order to track the planned trajectory (or stabilizing on it) and a great amount of control methodologies has been proposed to improve results in terms of stability and robustness and to take into account nonholonomy [19], [20], [3], [23], [22] and references therein. A general-

The authors are with the Institut National de Recherche en Informatique et en Automatique - INRIA-Sophia Antipolis(ICARE), address: 2004 Route des Lucioles BP 9306902 Sophia Antipolis Cedex - France, E-mail: \{FirstName.LastName\}@sophia.inria.fr. Alessandro Corrêa Victorino is supported by a doctoral fellowship from the Coordenação de Aperfeiçoamento de Pessoal de Nivel Superior - CAPES, Brazil, under Grant 2336/97-9. ized path tracking algorithm for nonholonomic mobile robots using transverse periodic functions is presented in [13]. In [6] feedback controller are derived to perform the trajectory tracking in a bounded error framework. At this level, there is no interaction with the environment during the robot motion and the feedback is performed using the proprioceptive (mainly, odometry) data.

In more realistic approaches, the perception of the environment by the robot's sensors is used at the motion control level, in order to control the interaction between the robot and its local environment. In such a way the robot motion is improved toward a safe navigation [9], [17], [4], [14]. In [21] the robot motion control is realized by combining visual servoing based tasks and repulsive potential fields associated to the obstacles in the environment.

In this context, we propose a new method lying on a robust sensor-based control approach which allows a complete exploration and mapping of an unknown indoor corridor-like environment. The setup is a mobile robot equipped with a laser scanning device which provides a planar cross section of the environment at a rate of $80 \mathrm{~ms}$ per revolution. Our exploration method is purely reactive (i.e. does not require a trajectory planning stage ) and guarantees a safe navigation in the free space of the environment. Moreover, as a straight property of the closed loop sensor-based control, we can guarantee that the robot moves during the exploration task with a bounded error which does not depend on the covered distance. The presentation of this methodology is structured in two complementary parts, Part-I and Part-II. In the Part-I, we introduce the navigation model and the sensor-based control framework lying on the range data provided by the laser scanning device. Three reactive navigation tasks and control laws are designed so that the robot is constrained to move on the Voronoï diagram of the environment without constructing it explicitly. It is known that a safe navigation requires a precise localization of the robot with respect to its environment and a well-adapted representation of the environment, these issues will be addressed in a second paper (PartII - Exploration, self-localization and map building). In this last part, we show the improvements obtained by embedding the problem of Simultaneous Localization 
and Mapping (SLAM) in the proposed sensor-based navigation framework. We also detail the modeling aspects of the environment based on an hybrid representation: metric and topological, which are simultaneously and incrementally constructed during the exploration of the environment. The robot is precisely localized in a set of metric maps associated to local well-defined places in the environment. A topological description of the environment allows a coarse localization when the robot navigates between two places.

The present paper addresses the Part-I of our sensorbased navigation methodology, and it is organized as follows. In the section II, we propose a model of the indoor environment well-adapted to support safe navigation tasks. This model, based on the Voronoï diagram, captures both the complete topology of the environment and the free space where the robot can navigate. From this model, we define three elementary navigation tasks : reach the nearest Voronoï branch, move along a branch and stop on a bifurcation point 1.

The section III deals with sensor-based control framework applied to our laser range finder sensor. After some general recalls on the sensor-based control framework, we derive a closed analytical form of the Jacobian of the sensor signal so-called Interaction $\mathrm{Ma}$ trix which relates the variation of the laser readings to the motion of the sensor. Finally, thanks to the task functions approach introduced by Samson et al [18], we show, in the section IV, it is possible to express the navigation tasks in terms of regulating to zero an output function only depending on the laser readings. A closed loop control scheme allows us to perform the navigation tasks robustly. We present in section $\mathrm{V}$ a stability analysis of the derived control laws as well as the robustness with regard to modeling errors and noisy measurement.

The experimental results of the control methodology applied to the robot navigation in indoor environment are presented in section VI. In section VII, we discuss some perspectives for future issues.

\section{NAVigation-Based Modeling of AN Indoor ENVIRONMENT}

We are interested in controlling the motion of the robot during its navigation in an indoor environment, so that the obstacles are avoided and the robot can access the whole free space during its exploration task. Already used by different authors [12], [15], [2], the Voronoï diagram is particularly well adapted to support navigation tasks.

The Voronoï diagram (VD), figure (1), (often called median axis or skeleton) is defined as the center of

\footnotetext{
${ }^{1}$ Some authors use the terms "meeting point" or "meet point"
}

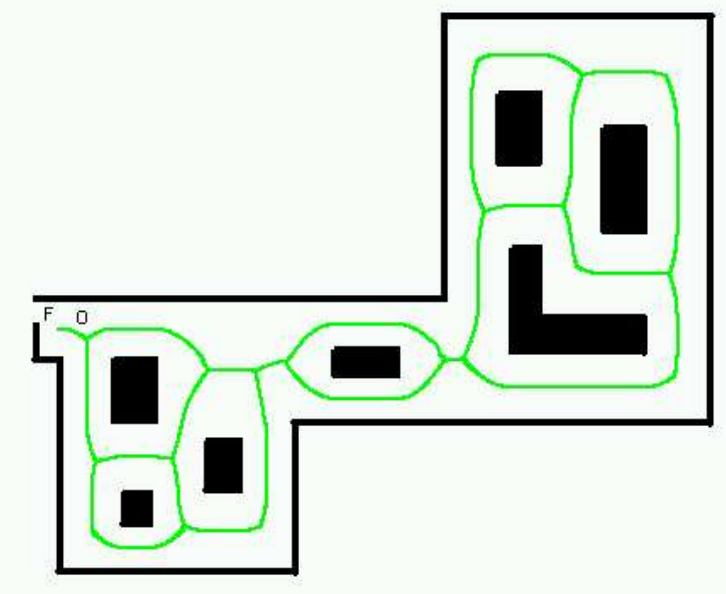

Fig. 1. An example of a simplified Voronoï diagram of an indoor environment. It is constituted by branches connected by bifurcation points.

the minimal balls (circles in 2D space) tangent to the obstacles. It defines a set of trajectories described by a graph where the branches are the locus of the centers of the minimal circles with two tangency points to the obstacles and the bifurcation points corresponds to the center of the minimal circles with more than two points of tangency. The VD satisfies the following properties:

- to be locally defined for each current location,

- to belong to the free space,

- to allow a complete exploration of the environment,

- to capture topology and accessibility of the map for a robot with a given size.

In terms of navigation tasks, moving on a Vorono:̈ branch can be viewed as a natural way to join two different places in an indoor environment. So, a complete exploration of an indoor environment can be performed by forcing the robot to move on the VD using the three following navigation tasks :

- $e_{1}$, reach the nearest Voronoï branch from any point of the free space and align the robot longitudinal axis on it,

- $e_{2}$, move ahead along a Voronoï branch,

- $e_{3}$, stop the robot on a Voronoï bifurcation point.

In the next section, we propose to implement such navigation tasks using a sensor-based control approach. We show that it is possible to perfectly achieve these tasks without building the VD explicitly. The problem of constructing a consistent model of the environment well-adapted to the execution of navigation tasks is addressed in Part-II of this work (submitted to publication), where a topological graph is generated by connecting the Voronoï bifurcation points of 


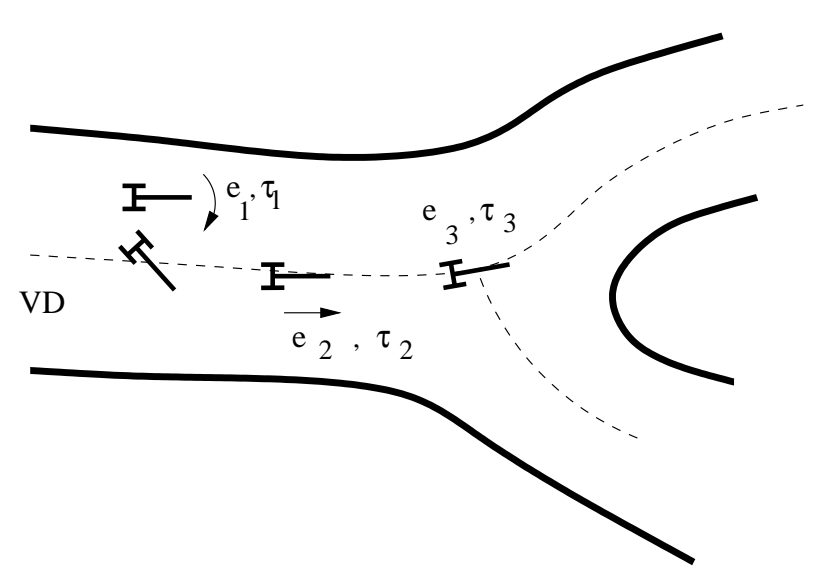

Fig. 2. The navigation tasks. $e_{1}, e_{2}$ and $e_{3}$ are respectively the tasks of stabilization on a branch, stabilization and motion along the branch and stabilization on a bifurcation point.

the environment found during the exploration task.

\section{ThE SENSOR-BASED CONTROL FRAMEWORK}

Here, we just present a brief overview of the general sensor-based control framework developed at Inria during the 90's. This framework can be applied for a large class of exteroceptive sensors including vision, force and range sensors. More details can be found in [18], [5], [16].

Let us consider a sensor $\mathcal{S}$ rigidly mounted on the robot that delivers a signal $s$. This signal is assumed only dependent of the relative pose $\bar{r}$ (position + attitude) between the sensor's frame and a reference frame attached to the environment $(s: \mathcal{C} \rightarrow \mathbb{R}$, where $\mathcal{C}$ is the configuration space for the frame linked to the sensor). This means that if the sensor is motionless with respect to the environment, then the signal remains constant. This assumption is valid in practice for a large class of sensors such as force, vision (geometric features) or range sensors. For an indoor mobile robot moving on flat floors, the configuration space $\mathcal{C}$ can be assimilated to the Special Euclidean group $\mathcal{S E}_{2}$ which is the group isomorphic to the group of homogeneous $3 \mathrm{X} 3$ matrices. $\bar{r}$ is an element of $\mathcal{S E}_{2}$ which can be parameterized by $(x, y, \theta)$. This hypothesis defines a local diffeomorphism between the configuration space $\mathcal{S E}_{2}$ and the output space of the sensor $\mathcal{S}$.

Moreover, $s e_{2}$, the Lie Algebra of $\mathcal{S E}_{2}$ (which is its tangent space at the identity), can be assimilated to the space of screws. The differential of $s$ is a linear mapping from $s e_{2}$ to $R$, it can be represented as a screw product :

$$
\dot{s}=\frac{\partial s}{\partial \bar{r}} \dot{\bar{r}}=\frac{\partial s}{\partial \bar{r}} T_{S E}=H \bullet T_{S E}
$$

where $T_{S E}$ is the velocity screw between the sensor and the environment frames. • denotes the screw product.

The matrix representation, called Interaction $\mathrm{Ma}$ trix, is the bilinear form associated to $H$, given by the relation :

$$
\dot{s}=L^{T} \cdot T_{S E} \quad \text { with } \quad L^{T}=H \cdot\left(\begin{array}{cc}
0 & I_{3} \\
I_{3} & 0
\end{array}\right)
$$

In a rough manner, $L^{T}$ can be viewed as a Jacobian relating the variation of the elementary sensor output $s$ to the relative displacement between the sensor and the environment.
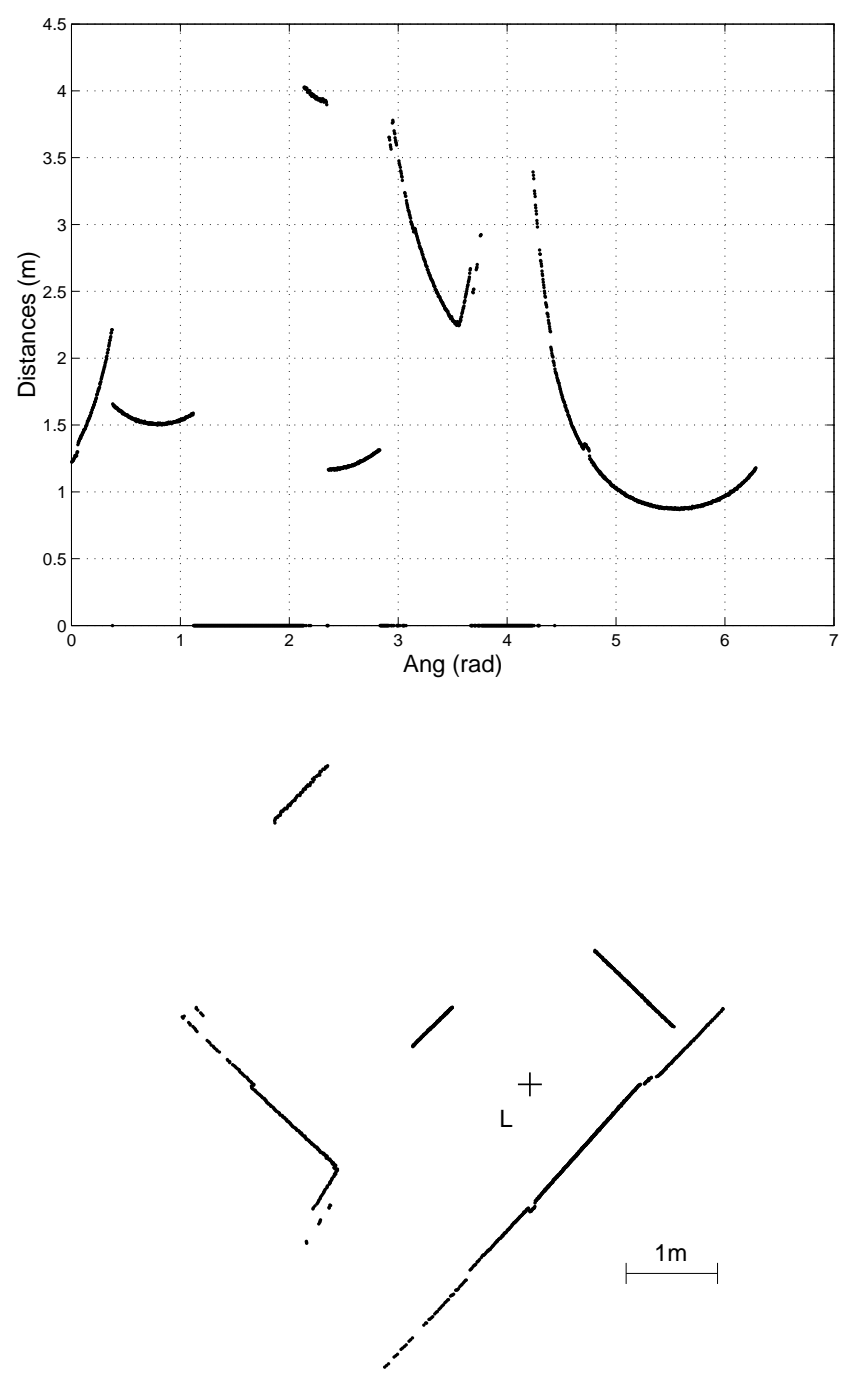

Fig. 3. Cross section of the environment represented in the laser frame. Top: Polar representation, Bottom: Cartesian representation

Let us now consider the application of this formalism to the case of a range sensor. The interaction with 
the environment is performed by a 2 -D laser scanning device which provides a planar cross section of the environment, shown in figure (3), considering the polar representation (figure 3 -top), the scan is represented by a set of distances and angles,

$$
S=\left\{\delta\left(\theta_{0}\right), \ldots, \delta\left(\theta_{i}\right), \ldots, \delta\left(\theta_{2 \pi}\right)\right\}
$$

were $\delta_{i}=\delta\left(\theta_{i}\right)$ is the distance from the origin of the controlled frame $(C)$, figure (4), to the nearest object $O_{i}$ at the angular position $\theta_{i}$, so we can choose as an elementary sensor signal $s_{i}=\delta_{i}$.

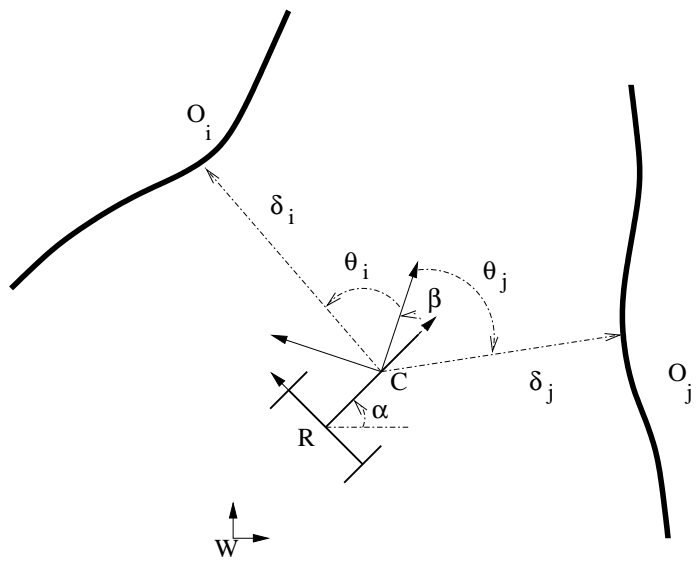

Fig. 4. The mobile robot $R$ carries a 2-D laser that measures the distances from the origin of the frame $C$ to the environment. The position of the frame $C$ in the reference frame $W$ is controlled by navigation control laws and then it is called "controlled frame". It's shown in the figure the distances $\delta\left(\theta_{i}\right), \delta\left(\theta_{j}\right)$ from the controlled frame $C$ to the objects $O_{i}$ and $O_{j}$, the robot's orientation in the world frame $W$ is $\alpha$ and the orientation of the controlled frame $C$ in $W$ is $\beta+\alpha$.

For simplicity, we suppose, in a first analysis, that the laser and the controlled frames are identical. Let us consider that the controlled frame $C$ is moving with a velocity screw $\overrightarrow{\tau_{C}}=\left(\overrightarrow{V_{C}}, \overrightarrow{\Omega_{C}}\right)$ where $\overrightarrow{V_{C}}$ and $\overrightarrow{\Omega_{C}}$ are respectively the translational and rotational velocities expressed in the frame $C$. Then, the variation of the sensor signal can be expressed, after some simple kinematic computation (see [16] for details on the derivation of this equation) :

$$
\begin{gathered}
\dot{\delta}_{i}=-\frac{1}{<\overrightarrow{n_{O}} \cdot \overrightarrow{n_{\theta_{i}}}>}\left[<\overrightarrow{n_{O}} \cdot \overrightarrow{V_{C}}>+\right. \\
\left.\delta_{i}<\left(\overrightarrow{n_{O}} \times \overrightarrow{n_{\theta_{i}}}\right) \cdot \Omega_{C}>\right]
\end{gathered}
$$

where $\langle.,$.$\rangle is the usual scalar product, \vec{n}_{\theta_{i}}$ is the unit vector in the direction of measurement and $\overrightarrow{n_{O}}$ is the unit vector normal to the surface of the object at the impact point of the laser beam. The equation (4) can be computed for all the points of impact in $S$ and constitutes the model of the interaction between the controlled frame $C$ and the environment.
Now, let us consider the peculiar point of impact $\left(\delta_{i}, \theta_{i}\right)$ such that $\delta_{i}$ is orthogonal to the surface of the object $O_{i}$ (figure (4)) so that,

$$
\overrightarrow{n_{O}}=-\vec{n}_{\theta_{i}}=\left[\begin{array}{ll}
-\cos \left(\theta_{i}\right) & -\sin \left(\theta_{i}\right)
\end{array}\right]
$$

¿From the interaction model in equation (4), the variation $\dot{\delta}_{i}$ can be evaluated as:

$$
\dot{\delta}_{i}=\left[\begin{array}{lll}
-\cos \theta_{i} & -\sin \theta_{i} & 0
\end{array}\right]\left[\begin{array}{c}
V_{x} \\
V_{y} \\
\Omega
\end{array}\right]
$$

where $-\pi<\theta_{i} \leq \pi$.

\section{The navigation functions}

Since the interaction screw defines a local mapping between the configuration space and the sensor output space, it becomes possible to relate the specification of a task in the configuration space to its specification in the sensor output space. Often, at the end-user level, a natural way to specify a local sensor-based task will be to define it in terms of geometrical constraints between the controlled sensor frame and a reference frame attached to a particular part of the environment. Using such specification rules, the navigation task $e_{1}, e_{2}$ and $e_{3}$ previously defined, can be expressed as constraints between the controlled sensor frame and a frame locally linked to the Voronoï diagram :

- $e_{1}$, reach the nearest Voronoï branch from any point of the free space is equivalent to constrain the origin of the controlled frame to converge to the nearest Voronoï branch and to align the $\mathrm{x}$-axis with the Voronoï branch.

- $e_{2}$, move ahead along a Voronoï branch is equivalent to constrain the $\mathrm{x}$-axis of the controlled frame to slide on the Voronoï branch.

- $e_{3}$, stop the robot on a Voronoï bifurcation point is equivalent to constrain the origin of the controlled frame to be stabilized on a Voronoï bifurcation point.

The next step will be to translate such tasks in terms of constraints on the sensor output signals shown in the figure 3-top. Let us consider the set of the minimal distances between the sensor and the obstacles modeled as a set of polygons:

$$
I_{\text {min }}=\left\{(\delta, \theta)_{0}, \ldots,(\delta, \theta)_{i}, \ldots,(\delta, \theta)_{n}\right\}
$$

each $(\delta, \theta)_{i}$ is a local minima of each piecewise continuous curve of the polar signature shown in the figure 3-top, where $\delta>0$ and $-\pi \leq \theta<\pi$. The set $I_{\text {min }}$ can be either extracted from $\mathcal{S}$ by performing a Hough transform or using a polygonal approximation algorithm. 
The navigation tasks are defined based on the following propositions:

Proposition 1: If the origin of the controlled frame is on a branch, then there exists two couples $\left\{(\delta, \theta)_{i},(\delta, \theta)_{j}\right\} \in I_{\min }$ such that,

$$
\left\{\begin{array}{l}
\delta_{i}=\delta_{j}=\min \left(\delta_{m}\right) \\
\theta_{i} \neq \theta_{j}
\end{array}\right.
$$

with $\delta_{m}>0, m \in\{1, \ldots, n\}$.

Proposition 2: The $\mathrm{x}$-axis of the controlled frame is collinear to the local direction of the Voronoï branch if, and only if, $\theta_{i}=-\theta_{j}$.

Proposition 3: If the origin of the controlled frame is on a bifurcation point then there exists at least three couples $\left\{(\delta, \theta)_{i},(\delta, \theta)_{j},(\delta, \theta)_{k}\right\} \in I_{\text {min }}$ such that,

$$
\left\{\begin{array}{l}
\delta_{i}=\delta_{j}=\delta_{k}=\min \left(\delta_{m}\right) \\
\theta_{i} \neq \theta_{j} \neq \theta_{k}
\end{array}\right.
$$

with $\delta_{m}>0, m \in\{1, \ldots, n\}$.

Based on the task function approach introduced by Samson [18], we can define a robot task as a regulation of an output function $\underline{e}(\bar{r}, t)$ directly expressed in the sensor output space $\mathcal{S}$. We consider that the task is perfectly achieved during the time interval $[0, T]$ iff : $\underline{e}(\bar{r}, t)=0, \forall t \in[0, T]$. The Jacobian $L^{T}=\frac{\partial e}{\partial \bar{r}}$ fully characterizes the local mapping between the sensor space $\mathcal{S}$ and the configuration space $\mathcal{C}$ and the $\operatorname{rank}\left(L^{T}\right)$ determines the dimension of the subspace of $\mathcal{C}$ controlled by the task function.

Lying on the propositions above, we can now design the navigation functions.

Navigation function $e_{1}$ : Reach a Voronoï branch

Based on the propositions 1 and 2 the navigation function $e_{1}$ is defined as:

$$
e_{1}(r)=\left(\begin{array}{c}
\delta_{1}-\delta_{2} \\
\frac{\theta_{1}+\theta_{2}}{2}
\end{array}\right)
$$

where the two laser measurements $\left(\delta_{1}, \theta_{1}\right)$ and $\left(\delta_{2}, \theta_{2}\right)$ are extracted from the set of minimal distances $I_{\text {min }}$ so that the distances $\delta_{1}$ and $\delta_{2}$ are smaller than all other distances $\delta_{k}$ in $I_{\text {min }}, k \neq 1$ and $k \neq 2$.

The variation of $e_{1}$ is obtained taking the derivative of (10) and from the interaction model described in the equation (6):

$$
\dot{e}_{1}=L^{T}\left[\begin{array}{c}
V_{x} \\
V_{y} \\
\Omega
\end{array}\right]
$$

where :

$$
L^{T}=\left[\begin{array}{ccc}
\left(c\left(\theta_{2}\right)-c\left(\theta_{1}\right)\right) & \left(s\left(\theta_{2}\right)-s\left(\theta_{1}\right)\right) & 0 \\
0 & 0 & -1
\end{array}\right]
$$

with $c\left(\theta_{i}\right)=\cos \left(\theta_{i}\right)$ and $s\left(\theta_{i}\right)=\sin \left(\theta_{i}\right) . \quad L^{T}$ is the interaction matrix.

Let us now consider the case where the task $e_{1}$ is perfectly achieved (i.e. $e_{1}=0 \rightarrow\left\{\delta_{1}=\delta_{2}, \theta_{1}=\right.$ $\left.\left.-\theta_{2}=\theta\right\}\right)$, then $\dot{e}_{1}=0$ and the equation (11) yields :

$$
0=L_{\left(e_{1}=0\right)}^{T}\left[\begin{array}{c}
V_{x} \\
V_{y} \\
\Omega
\end{array}\right]=L_{\left(e_{1}=0\right)}^{T} \tau^{\star}
$$

where:

$$
L_{\left(e_{1}=0\right)}^{T}=\left[\begin{array}{ccc}
0 & 2 \sin (\theta) & 0 \\
0 & 0 & -1
\end{array}\right]
$$

The dimension of the space spanned by the interaction matrix (14) is two, $\operatorname{Rank}\left(L_{\left(e_{1}=0\right)}^{T}\right)=2$ and its null space is spanned by,

$$
\operatorname{Ker}\left(L_{\left(e_{1}=0\right)}^{T}\right)=\left[\begin{array}{lll}
1 & 0 & 0
\end{array}\right]
$$

That means that only 2 degrees of freedom are constrained by the execution of the task $e_{1}$. There exist then a motion of the controlled frame, $\tau^{\star} \neq 0$ in (13), not constrained by the task $e_{1}$, that belongs to the null space of $L_{\left(e_{1}=0\right)}^{T}$. Equation (15) indicates that such a motion which does not affect the realization of the task $e_{1}$, corresponds to the displacement along the $\mathrm{x}$-axis of the controlled frame. This unconstrained degree of freedom will be used by a secondary task enabling the displacement along the Voronoï branch.

Navigation function $e_{2}$ : Move ahead along the Voronoï branch

A second task $e_{2}$ is then designed that corresponds to a motion of the controlled frame onto the null space of $L^{T} \cdot e_{2}$ is designed so that the robot moves with a given constant velocity $v$ along the Voronoï diagram.

Let us consider the following motion constraint,

$$
F=X(t)-X_{0}-V_{d} \cdot t=0
$$

that consists in a movement with a constant velocity $V_{d}=\left[v \cos \left(\theta_{c}\right) v \sin \left(\theta_{c}\right)\right]$ of the controlled frame $C$ (figure 4) along the $\mathrm{VD}$, where $X=\left[\begin{array}{ll}x y & y\end{array}\right]^{T}$ is the position of the controlled frame w.r.t. the reference frame. The second task is then defined as a cost function $e_{2}=\frac{1}{2} F^{T} F$ to be minimized under the constraint that $e_{1}=0$. The gradient of $e_{2}$, expressed in the reference frame, is given by $G_{s}=\frac{\partial e_{2}}{\partial X}=F$ and it is projected into the controlled frame by,

$$
g_{s}=R_{W L}^{T} G_{s}
$$

where $R_{W L}$ is the rotation matrix between the controlled frame $C$ and the reference frame $W$ as shown in the figure (4). 
The velocity $V_{d}=\left[\begin{array}{ll}v_{d x} & v_{d y}\end{array}\right]^{T}$ is smoothed by a linear filter such that,

$$
V_{d}^{\prime}=\alpha_{1}\left(X(t)-X_{d}(t)\right)+V_{d}
$$

where $X_{d}(t)$ is represented in the discrete version as,

$X_{d}(k+1)=\left(1-\beta_{1}\right) X_{d}(k)+\beta_{1} X(k)+V_{d}^{\prime}(k) \Delta t$

$\alpha_{1}>0$ and $\beta_{1}>0$ are tuning parameters.

We use the formalism of the redundant tasks described in [18] to consider the tasks $e_{1}$, defined in the equation (10), and $e_{2}$ together in a global task $e_{\text {branch }}$ :

$$
e_{\text {branch }}=W^{+} C e_{1}+\alpha_{t}\left(I_{3}-W^{+} W\right) g_{s}^{T}
$$

where:

- $e_{\text {branch }}$ is an orthogonal combination of $e_{1}$ and $e_{2}$.

- $\alpha_{t}$ is a positive weight tuning between the tasks $e_{1}$ and $e_{2} ;\left(\alpha_{t} \leq 1\right)$.

- $W$ is a $(m \times n)$ full-rank matrix, so that $\operatorname{Ker}(W)=$ $\operatorname{Ker}\left(L^{T}\right), m$ is the dimension of $e_{1}$ and $n$ the number of degrees of freedom of the controlled frame.

- $\left(I_{3}-W^{+} W\right)$ is the orthogonal projection operator onto the null space of $W, I_{3}$ represents the $(3 \times 3)$ identity matrix.

- $W^{+}$pseudo-inverse of $W$.

- $C$ is a combination matrix, so that with $C L^{T} W^{+}>$ 0 the regularity of $e_{1}$ is guaranteed. A good choice is $C=W L^{T^{+}},[18]$.

- $L^{T}$ is the interaction matrix described in the equation (12).

- $g_{s}$ is defined in the equation (17).

Navigation function $e_{3}$ : Stop on a Voronoï bifurcation point

Based on the propositions 2 and 3, the navigation function $e_{3}$ is defined as:

$$
e_{3}(r)=\left(\begin{array}{c}
\delta_{1}-\delta_{2} \\
\delta_{1}-\delta_{3} \\
\frac{\theta_{1}+\theta_{2}}{2}
\end{array}\right)
$$

where $\left\{(\delta, \theta)_{1},(\delta, \theta)_{2},(\delta, \theta)_{3}\right\} \in I_{\min }$ so that $\delta_{1}, \delta_{2}$ and $\delta_{3}$ are smaller than all other distances $\delta_{k}$ in $I_{\text {min }}, k \notin$ $\{1,2,3\}$.

Applying the interaction model from equation (6) yields :

$$
\dot{e}_{3}=L^{T}\left[\begin{array}{c}
V_{x} \\
V_{y} \\
\Omega
\end{array}\right]
$$

where :

$$
L^{T}=\left[\begin{array}{ccl}
\left(c\left(\theta_{2}\right)-c\left(\theta_{1}\right)\right) & \left(s\left(\theta_{2}\right)-s\left(\theta_{1}\right)\right) & 0 \\
\left(c\left(\theta_{3}\right)-c\left(\theta_{1}\right)\right) & \left(s\left(\theta_{3}\right)-s\left(\theta_{1}\right)\right) & 0 \\
0 & 0 & -1
\end{array}\right]
$$

with $c\left(\theta_{i}\right)=\cos \left(\theta_{i}\right)$ and $s\left(\theta_{i}\right)=\sin \left(\theta_{i}\right)$.

Considering the null space of the matrix $L^{T}$ :

$$
\operatorname{Ker}\left(L^{T}\right)=\{\emptyset\}
$$

The stabilization task $e_{3}$ constraints the whole set of the robot configurations and there is no degree of freedom to be exploited in a secondary task. Then from equation (20) with $g_{s}^{T}=0$,

$$
e_{B P}=W^{+} C e_{3}
$$

- $W$ is chosen $W=I_{3}$, so that $\operatorname{Ker}(W)=\operatorname{Ker}\left(L^{T}\right)$. - $C=W L^{-T}$ is the combination matrix.

and,

$$
e_{B P}=L^{-T} e_{3}
$$

\section{The Control Laws}

This section is dedicated to the design of the feedback control laws able to realize the navigation functions described in the section III.

Let $e$ be a generic form of a navigation task (i.e. $e_{\text {branch }}$ in (20), or $e_{B P}$ in (26)). Computing the derivative of $e$ which is $r$ and time dependent, yields :

$$
\dot{e}=\frac{\partial e}{\partial r} \tau_{C}+\frac{\partial e}{\partial t}
$$

Assuming an exponential decay $\dot{e}=-\lambda e$ of the task function with a convergence rate tuned by the gain $\lambda$, the desired velocity screw of the controlled frame is given by,

$$
\tau_{C}=\left(\frac{\partial e}{\partial r}\right)^{-1}\left(-\lambda e-\frac{\partial e}{\partial t}\right)
$$

Let us now apply equation (28) to the task function $e_{\text {branch }}$. From the equation (20), we get :

$$
\frac{\partial e_{\text {branch }}}{\partial t}=W^{+} C \frac{\partial e_{1}}{\partial t}+\alpha_{t}\left(I_{3}-W^{+} W\right) \frac{\partial g_{s}^{T}}{\partial t}
$$

The term $\frac{\partial e_{1}}{\partial t}$ in this equation accounts an eventual motion of the obstacle frame which, in our case, is static, so $\frac{\partial e_{1}}{\partial t}=0$. We can consider $\frac{\partial e_{b r a n c h}}{\partial r}=I_{3}$ for stability and simplicity purposes and the desired velocity control input $\tau_{C}$, is given by:

$$
\tau_{C}=-\lambda e_{\text {branch }}-\alpha_{t}\left(I_{3}-W^{+} W\right) \frac{\partial g_{s}^{T}}{\partial t}
$$

$W$ is chosen as,

$$
W=\left[\begin{array}{ll}
0 & I_{2}
\end{array}\right]
$$


Finally, the feedback control input for the navigation task along the branch $e_{\text {branch }}$ is done by:

$\tau_{C}=-\lambda e_{\text {branch }}+\alpha_{t}\left[\begin{array}{c}v_{d x} \cos \left(\theta_{C}\right)+v_{d y} \sin \left(\theta_{C}\right) \\ 0 \\ 0\end{array}\right]$

where $\theta_{C}=\beta+\alpha$ as seen in figure (4). Let us note that the angle $\beta$ must be introduced in order to control the sensor frame $C$ when the sensor is carried out by a two wheels-driven non holonomic mobile robot. As a matter of fact, it is not possible to fully control the 3 -dimensions space $\left\{x, y, \theta_{C}\right\}$ uniquely from the 2 control inputs corresponding to a two wheels-driven mobile robot. To over-pass this problem, a classical solution consists to mount the sensor on a pan motorized axis. In our case, rather than using an extra motorized axis, we define the angle $\beta$ which can be viewed as a virtual degree of freedom (in fact an offset angle added on the laser readings). It was considered as part of the robot state to circumvent the nonholonomic constraint that is intrinsic to the kinematic model of an unicycle mobile robot. The extended robot state is then $\{x, y, \alpha, \beta\}$, figure (4).

In the case of the navigation task $e_{B P}$, stabilization on a bifurcation point, $g_{s}^{T}=0$ and the feedback control input is done by:

$$
\tau_{C}=-\lambda e_{B P}
$$

The control inputs (32) and (33) are defined into the controlled frame $C$. They will be applied to the two wheels-driven mobile robot after the transformation :

$$
u=\left(\begin{array}{c}
v \\
\omega \\
\dot{\beta}
\end{array}\right)=J_{R}^{-1} \tau_{C}
$$

where $v=\dot{x} \cos (\alpha)+\dot{y} \sin (\alpha)$ is the heading speed of the mobile platform, $\omega$ is the angular velocity and $J_{R}$ is the Jacobian matrix between the controlled frame $C$ and the robot frame $R$, figure (4). $J_{R}^{-1}$ is given by,

$$
J_{R}^{-1}=\left(\begin{array}{ccc}
\cos (\beta) & -\sin (\beta) & 0 \\
\frac{\sin (\beta)}{d} & \frac{\cos (\beta)}{d} & 0 \\
\frac{-\sin (\beta)}{d} & \frac{-\cos (\beta)}{d} & 1
\end{array}\right)
$$

where $d>0$ is the $\mathrm{x}$-coordinate of the controlled frame $C$ into the robot frame $R$, as represented in figure 5 .

\section{Stability and Robustness issues}

We present in this section the necessary conditions for the stability and robustness of the control laws considering modeling errors on the robot and noisy measurements provided by the laser. The model of the real

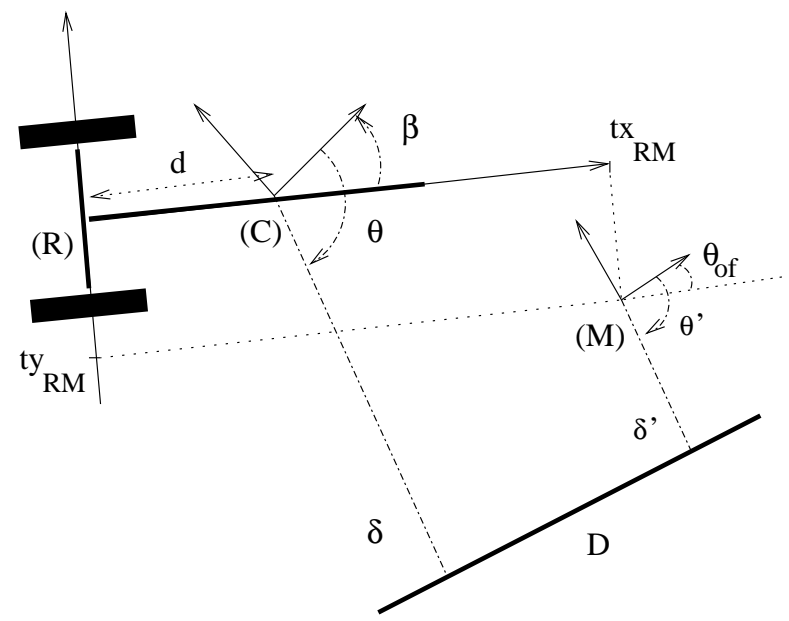

Fig. 5. The configuration of the control system. The laser measurements $\left(\theta^{\prime}, \delta^{\prime}\right)$ are acquired in the frame $M$ fixed at a point $\left(t x_{R M} \quad t y_{R M}\right)$ with an orientation $\theta_{o f}$ in the robot frame $R$. These measurements are projected as $(\theta, \delta)$ into the frame $C$ where the control inputs are computed. The projection from $M$ into $C$ is done using an uncertain rigid transformation between $M$ and $R$ experimentally identified and represented by a translation $\hat{T}_{R M}=\left(\begin{array}{ll}\hat{t}_{x_{R M}} & \hat{t}_{y_{R M}}\end{array}\right)^{T}$ and a rotation $\hat{\theta}_{o f}$ and the transformation between $R$ and $C$ characterized by the distance $d$ and the orientation $\beta$ perfectly known.

setup is described in the figure 5 , as an extension of the figure 4 , where it is shown:

- The robot frame $R$ fixed on the robot basis.

- The point $x_{c}=\left[\begin{array}{ll}d & 0\end{array}\right]^{T}, d>0$, fixed on the frame $R$.

- The controlled frame $C$ with the origin in $x_{c}$ in the frame $R$ and with a rotation $\beta$ with respect to $R$. The interaction matrices and the control laws are calculated in $C$.

- The sensor frame $M$ rigidly fixed on the robot where the laser measurements are acquired. The rigid transformation between $M$ and $R$ is given by a translation $T_{R M}=\left[\begin{array}{ll}t x_{R M} & t y_{R M}\end{array}\right]^{T}$ and a rotation $\theta_{o f}$ as indicated in the figure 5 . These values are identified experimentally from the real platform.

Two sources of uncertainties are considered in the stability analysis :

- The noise associated to the laser sensor and propagated by the segmentation process of the lines $\left(\theta^{\prime}, \delta^{\prime}\right)=\left(\theta^{\prime \prime}, \delta^{\prime \prime}\right)+\nu_{(\theta, \delta)}$. The error $\nu_{(\theta, \delta)}$ in the real laser readings $\left(\theta^{\prime \prime}, \delta^{\prime \prime}\right)$ is a characteristic of the physical sensor.

- The uncertainty in the rigid transformation $\left(\hat{T}_{R M}, \hat{\theta}_{o f}\right)$ which is identified experimentally and provides an approximation of the real transformation $\left(T_{R M}, \theta_{o f}\right)$.

Let us consider as data inputs the parameters of the segmented lines computed from the laser scan. The line $D$ in the figure 5 is parameterized by $\left(\theta^{\prime}, \delta^{\prime}\right)$ in the 
laser frame $M$ and by $(\theta, \delta)$ in the controlled frame $C$. Considering the uncertain transformation $\left(\hat{T}_{R M}, \hat{\theta}_{o f}\right)$ then $\left(\theta^{\prime}, \delta^{\prime}\right)$ will be represented as $(\hat{\theta}, \hat{\delta})$ in the frame $C$.

After stating the differential equation that represents the real behavior of the closed loop control system, the following issues are carried out by the analysis:

- The conditions for the existence and uniqueness of an equilibrium solution.

- The stability and robustness analysis with regard to the bounds of the errors $\left(\hat{T}_{R M}-T_{R M}\right)$ and $\left(\hat{\theta}_{o f}-\theta_{o f}\right)$.

We consider both the cases of stabilization on the VD branches and the stabilization on the VD bifurcation points.

A. Stability analysis for the stabilization on the VD branches

The stabilization of the controlled frame $C$ on a VD branch and the laser measurements are recalled in the figure 6 . Let us consider the global task $e_{\text {branch }}$ de-

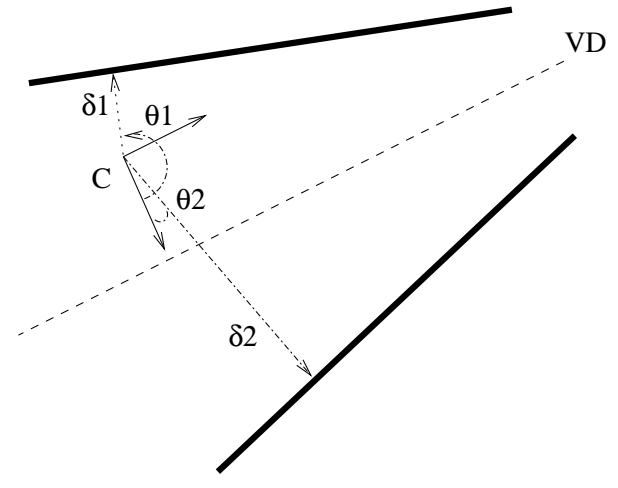

Fig. 6. The stabilization of the robot at a VD branch. Only the controlled frame $C$ is shown for simplicity. $\left(\theta_{1}, \delta_{1}\right)$ and $\left(\theta_{2}, \delta_{2}\right)$ as indicated in the figure are the minimal distances and orientations from the origin of $C$ to the two objects represented by the lines. $C$ is stabilized at the VD from an initial position with the regulation of the task $e_{1}$ in (10).

scribed in (20) with $\alpha_{t}=0$ :

$$
e_{\text {branch }}=W^{+} W L^{T^{+}} e_{1}
$$

The control law $\tau_{C}$ in (32) is rewritten as:

$$
\tau_{C}=-\lambda W^{+} W L^{T^{+}} e_{1}
$$

The task $e_{1}$ is given in (10) as:

$$
e_{1}=\left(\begin{array}{c}
\delta_{1}-\delta_{2} \\
\frac{\theta_{1}+\theta_{2}}{2}
\end{array}\right)
$$

where $\left(\theta_{1}, \delta_{1}\right)$ and $\left(\theta_{2}, \delta_{2}\right)$ are as shown in the figure 6 . The matrices $L^{T}$ and $W$ are defined in section III and recalled hereafter:

$$
L^{T}=\left[\begin{array}{ccc}
\left(c\left(\theta_{2}\right)-c\left(\theta_{1}\right)\right) & \left(s\left(\theta_{2}\right)-s\left(\theta_{1}\right)\right) & 0 \\
0 & 0 & -1
\end{array}\right]
$$

with $c\left(\theta_{i}\right)=\cos \left(\theta_{i}\right)$ and $s\left(\theta_{i}\right)=\sin \left(\theta_{i}\right)$. Recalling that $W$ is a matrix defined so that $\operatorname{Ker}(W)=\operatorname{Ker}\left(L^{T}\right)$, in equation (20), then $W=L^{T}$ without loss of generality.

As the transformation $\left(T_{R M}, \theta_{o f}\right)$ is not known exactly and measurements are corrupted by noise then the control input used into the control loop is calculated as:

$$
\tau_{C}=-\lambda \hat{W}^{+} \hat{W} \hat{L}^{T} \hat{e}_{1}
$$

The matrices $\hat{L^{T}}, \hat{W}$ and the task $\hat{e}_{1}$ are calculated from the laser readings $(\hat{\theta}, \hat{\delta})$ in the frame $C$.

Considering the derivative $\dot{e}_{1}$ in equation (11), rewritten here,

$$
\dot{e}_{1}=L^{T}\left[\begin{array}{c}
V_{x} \\
V_{y} \\
\Omega
\end{array}\right]=L^{T} \tau_{C}
$$

the differential equation that represents the closed loop response of the system is written taken the equation (40) in (41):

$$
\dot{e}_{1}=-\lambda L^{T} \hat{W}^{+} \hat{W} \hat{L}^{T}{ }^{+} \hat{e}_{1}
$$

As it is shown in the Appendix A, the modeling errors act as an additive bias on the task function $e_{1}$, so :

$$
\hat{\dot{e}}_{1}=\dot{e}_{1}
$$

and the equation (42) can be rewritten as,

$$
\begin{gathered}
\hat{\dot{e}}_{1}=-\lambda L^{T} \hat{W}^{+} \hat{W} \hat{L}^{T^{+}} \hat{e}_{1} \\
\hat{\dot{e}}_{1}=-\lambda A_{1} \hat{e}_{1}
\end{gathered}
$$

where $\lambda>0$.

The stability analysis is then done regarding to the positivity of the matrix $A_{1}(\theta, \delta)$ which is written from (44) as:

$$
A_{1}=\left[\begin{array}{cc}
\cos \left(\frac{\theta_{1}+\theta_{2}}{2}-\frac{\hat{\theta}_{1}+\hat{\theta}_{2}}{2}\right) \frac{\sin \left(\frac{\theta_{1}-\theta_{2}}{2}\right)}{\sin \left(\frac{\hat{\theta}_{1}-\hat{\theta}_{2}}{2}\right)} & 0 \\
0 & 1
\end{array}\right]
$$

Then, the theorem 1 gives the necessary conditions for the existence and uniqueness of an equilibrium solution of (44) and the sufficient conditions on the bounds of the errors $\left(\hat{T}_{R M}-T_{R M}\right)$ and $\left(\hat{\theta}_{o f}-\theta_{o f}\right)$ for the stability and convergence to such equilibrium solution. 


\section{Theorem 1:}

(i)(Existence and uniqueness) If the configuration is not degenerated so that $\theta_{1} \neq \theta_{2}$ and the VD branch exists, then the control system (44) has a unique equilibrium solution $\hat{e}_{1}=0$ if, and only if,

$$
\left|\hat{\theta}_{o f}-\theta_{o f}\right| \neq \frac{\pi}{2}
$$

(ii) (Global stability) The system (44) converges asymptotically from a given initial position to the equilibrium solution if, and only if,

$$
\left|\hat{\theta}_{o f}-\theta_{o f}\right|<\frac{\pi}{2}
$$

\section{Proof 1:}

(i) The solution $\hat{e}_{1}=0$ of $A_{1} \cdot \hat{e}_{1}=0$ exists and it is unique if $\operatorname{det}\left(A_{1}\right) \neq 0$. The determinant of $A_{1}$ is written as

$$
\operatorname{det}\left(A_{1}\right)=\cos \left(\frac{\theta_{1}+\theta_{2}}{2}-\frac{\hat{\theta}_{1}+\hat{\theta}_{2}}{2}\right) \cdot \frac{\sin \left(\frac{\theta_{1}-\theta_{2}}{2}\right)}{\sin \left(\frac{\hat{\theta}_{1}-\hat{\theta}_{2}}{2}\right)}
$$

and,

(i-1) the configuration of the system, figure 6 , is not degenerated so that $\theta_{1} \neq \theta_{2}$ and the VD branch exists, then

$$
\sin \left(\frac{\theta_{1}-\theta_{2}}{2}\right) \neq 0
$$

(i-2) with the relations between $(\theta, \delta)$ and $(\hat{\theta}, \hat{\delta})$ derived in the Appendix $\mathrm{A}$ and rewritten below:

$$
\begin{aligned}
& \frac{\theta_{1}-\theta_{2}}{2}=\frac{\hat{\theta}_{1}-\hat{\theta}_{2}}{2} \\
& \frac{\theta_{1}+\theta_{2}}{2}-\frac{\hat{\theta}_{1}+\hat{\theta}_{2}}{2}=\theta_{o f}-\hat{\theta}_{o f}
\end{aligned}
$$

so,

$$
\sin \left(\frac{\theta_{1}-\theta_{2}}{2}\right)=\sin \left(\frac{\hat{\theta}_{1}-\hat{\theta}_{2}}{2}\right) \neq 0
$$

Then from (49) and (50) the condition $\operatorname{det}\left(A_{1}\right) \neq 0$ is verified if, and only if,

$$
\left|\hat{\theta}_{o f}-\theta_{o f}\right| \neq \frac{\pi}{2}
$$

(ii) It is well known that the convergence of the system (44) is asymptotic if $A_{1}$ is positive definite, $A_{1}>0$ $\left(A_{1}>0\right.$ in the sense that $\left.x^{T} A_{1} x>0 \forall x \neq 0\right) . A_{1}$ is positive-defined if all of its eigenvalues are positives. The eigenvalues of $A_{1}$ are $\zeta_{1}$ and $\zeta_{2}$ :

$$
\begin{aligned}
& \zeta_{1}=1 \\
& \zeta_{2}=\cos \left(\frac{\theta_{1}+\theta_{2}}{2}-\frac{\hat{\theta}_{1}+\hat{\theta}_{2}}{2}\right) \cdot \frac{\sin \left(\frac{\theta_{1}-\theta_{2}}{2}\right)}{\sin \left(\frac{\hat{\theta}_{1}-\hat{\theta}_{2}}{2}\right)}
\end{aligned}
$$

Then with the relations (50) and (51) the condition $\zeta_{2}>0$ is verified if, and only if,

$$
\left|\hat{\theta}_{o f}-\theta_{o f}\right|<\frac{\pi}{2}
$$

Remark 1: The stability of the closed loop control system (44) is not dependent on the modeling errors on the rigid translation between the sensor frame $M$ and the robot basis frame $R$ given by $\left(\hat{T}_{R M}-T_{R M}\right)$. Only the error $\left(\hat{\theta}_{o f}-\theta_{o f}\right)$ affects the stability of the system.

Remark 2: The noise on the measurements $\left(\theta^{\prime}, \delta^{\prime}\right)$, $\nu_{(\theta, \delta)}$, is not considered in the stability analysis above. In practice, the errors due to the noisy measurements will be negligible with regard to the errors on the geometric model. So, the large domain of convergence resulting from the Theorem 1 credits the hypothesis that the noise on the measurements does not affect the stability of the system.

Remark 3: It is shown in the Appendix A that when the measured task $\hat{e}_{1}$ in the system (44) is regulated to the origin, $\hat{e}_{1} \rightarrow 0$, then the real task $e_{1}$ is stabilized to an offset value which is a function of the model errors $\left(\hat{\theta}_{o f}-\theta_{o f}\right)$ and $\left(\hat{T}_{R M}-T_{R M}\right), e_{1} \rightarrow \operatorname{bias}\left(\hat{\theta}_{o f}, \hat{T}_{R M}\right)$.

\section{B. Stability analysis on the stabilization to a bifurca- tion point}

The stabilization task at the neighborhood of a bifurcation point given in (26) is rewritten below:

$$
e_{B P}=L^{-T} e_{3}
$$

where $e_{3}$ is given in (21) as:

$$
e_{3}=\left(\begin{array}{c}
\delta_{1}-\delta_{2} \\
\delta_{1}-\delta_{3} \\
\frac{\theta_{1}+\theta_{2}}{2}
\end{array}\right)
$$

and the interaction matrix $L^{T}$ in $(23)$

$$
L^{T}=\left[\begin{array}{ccl}
\left(c\left(\theta_{2}\right)-c\left(\theta_{1}\right)\right) & \left(s\left(\theta_{2}\right)-s\left(\theta_{1}\right)\right) & 0 \\
\left(c\left(\theta_{3}\right)-c\left(\theta_{1}\right)\right) & \left(s\left(\theta_{3}\right)-s\left(\theta_{1}\right)\right) & 0 \\
0 & 0 & -1
\end{array}\right]
$$

with $c\left(\theta_{i}\right)=\cos \left(\theta_{i}\right)$ and $s\left(\theta_{i}\right)=\sin \left(\theta_{i}\right)$. The control law used in the stabilization is done in (33) and rewritten as:

$$
\tau_{C}=-\lambda L^{-T} e_{3}
$$

As described in section $\mathrm{V}$-A the control input used in the closed control loop calculated from corrupted measurements is written as:

$$
\tau_{C}=-\lambda \hat{L}^{-T} \hat{e}_{3}
$$


where $\hat{L}$ and $\hat{e}_{3}$ are calculated from measures $(\hat{\theta}, \hat{\delta})$.

Considering the derivative of the task $e_{3}$, presented in $(22)$ :

$$
\dot{e}_{3}=L^{T}\left[\begin{array}{c}
V_{x} \\
V_{y} \\
\Omega
\end{array}\right]=L^{T} \tau_{C}
$$

then the differential equation that represents the response of the closed loop system is given by:

$$
\dot{e}_{3}=-\lambda L^{T} \hat{L}^{-T} \hat{e}_{3}
$$

The relation between $\hat{e}_{3}$ and $e_{3}$ is established in the same way as the relation between $\hat{e}_{1}$ and $e_{1}$ presented in the Appendix A, so that,

$$
\hat{\dot{e}}_{3}=\dot{e}_{3}
$$

and (61) is rewritten as,

$$
\begin{gathered}
\hat{\dot{e}}_{3}=-\lambda L^{T} \hat{L}^{-T} \hat{e}_{3} \\
\hat{\dot{e}}_{3}=-\lambda A_{3} \hat{e}_{3}
\end{gathered}
$$

where $\lambda>0$.

The existence and uniqueness of an equilibrium solution of the closed loop system (63) is directly related to the existence of a bifurcation point associated to the environment. Then the system (63) has a unique equilibrium solution $\hat{e}_{3}=0$ if there exists a bifurcation point, that is $\theta_{i} \neq \theta_{j} \forall i \neq j ; i, j \in\{1,2,3\}$. The stability and robustness at the convergence with respect to the errors on the model are given with the results of the Theorem 2.

Theorem 2: If there exists a bifurcation point and the control law (59) is applied then the convergence to the equilibrium solution $\hat{e}_{3}=0$ of the closed loop control system (63) is global asymptotically stable if, and only if,

$$
\left|\hat{\theta}_{o f}-\theta_{o f}\right| \neq \frac{\pi}{2}
$$

The proof of the Theorem 2 is given in the Appendix B.

The results of the Theorems 1 and 2 show that even with a large range on the modeling errors $\left|\hat{\theta}_{o f}-\theta_{o f}\right| \neq$ $\frac{\pi}{2}$ the control systems (44) and (63) are global asymptotically stable. This fact shows the robustness of the control laws (37) and (59) designed in section IV.

The important result of the stability analysis is the following : assuming the existence of a Voronoï Diagram characteristic of the real physical environment, the sensor-based control laws using only local perception data ensure the robot convergence onto the Voronoï Diagram with a bounded error depending on the control gains $\lambda$ exclusively.

\section{EXPERIMENTAL RESULTS}

In this section, we present results validating the application of the control methodology derived for the safe navigation of a robot.

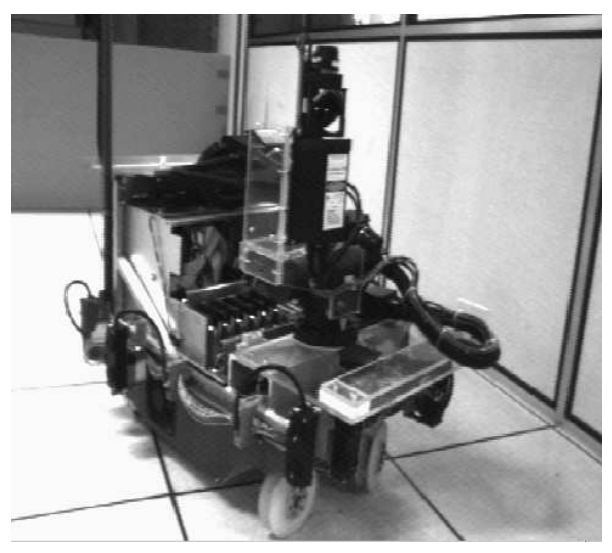

Fig. 7. The robot ANIS.

The test-bed is constituted with the mobile robot ANIS, figure 7, developed in our laboratory ${ }^{2}$. This robot is equipped with a $2 \mathrm{D}$-Laser range-finder with a scanning device that delivers 2000 points of measurements distributed in a 360 degrees scan at $80 \mathrm{~ms}$ rate.

The figure 8(top) shows an example of robot trajectory obtained using the sensor-based control laws designed in the section IV, in an indoor structured but unknown environment. The robot starts from a position marked $s$ in the figure, it reaches the Voronoï branch at the position marked $V D$ and moves along the branch using the control law defined in (32). The stabilization and the dispacement on the branch are performed using as feedback the minimal distances between the robot and objects $O_{1}$ and $O_{2}$. These minimums, $\left(\delta_{1}, \theta_{1}\right)$ and $\left(\delta_{2}, \theta_{2}\right)$, are extracted from the laser scans and tracked during the robot motion. In figure 9, it is illustrated the evolution of the distances (and orientations) between the instants $k$ (solid lines) and $(k+1)$ (dashed lines) during the navigation on the branch. The signals at time $(k+1)$ are extracted in windows predicted from the signals at the instant $k$.

As the robot is moving toward the object $O_{3}$, a third distance $\delta_{3}$ falls under a given threshold, illustrated as $D_{3 r d}$ in the figure 9 . Then a third window is initialized and associated to $\left(\delta_{3}, \theta_{3}\right)$ which is equally tracked. The controller switches on the control (33) and the robot is then stabilized to the correspondent bifurcation point $\mathrm{BP} 1$.

For leaving the point $\mathrm{BP} 1$, the robot calculates the possible exiting branches associated to this BP that are still unexplored based on the incoming branch and

${ }^{2}$ http://www-sop.inria.fr/icare/icare-fra.html 

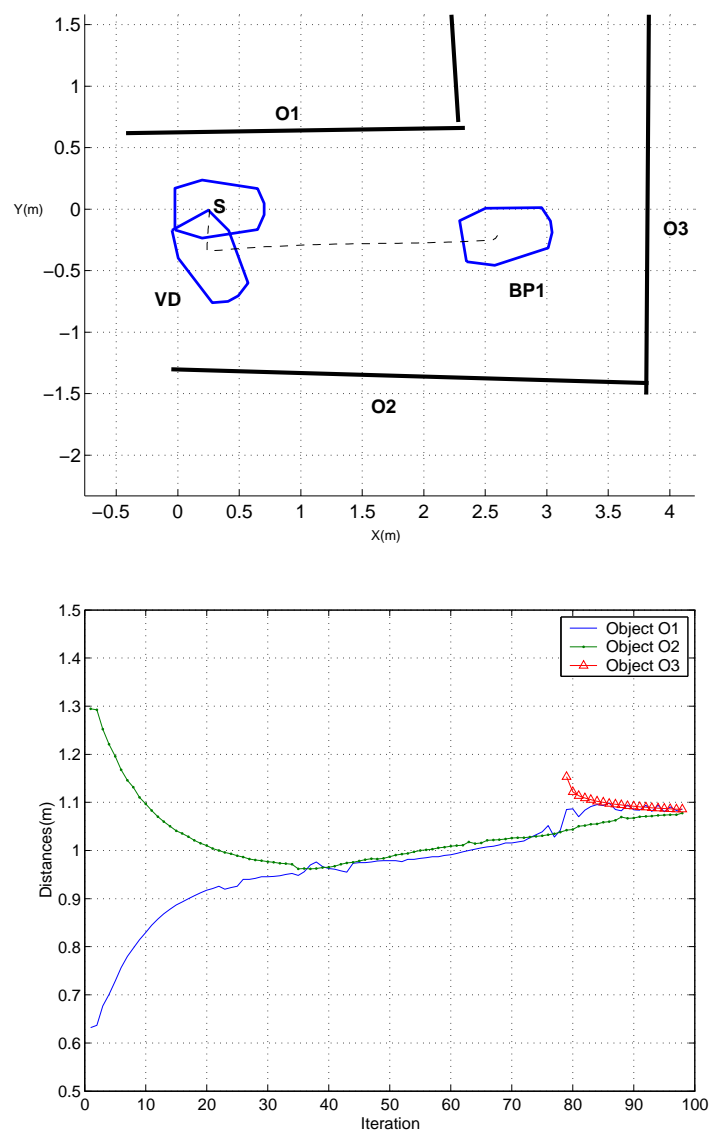

Fig. 8. (top) The application of the control laws (32) and (33). (bottom) The evolution of the signals in the stabilization at the branch, movement along the branch and stabilization at a bifurcation point.

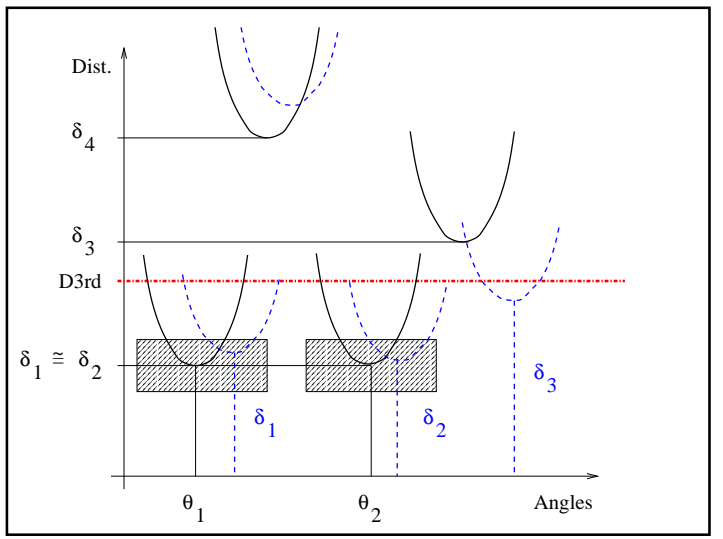

Fig. 9. Distance tracking process on the occultations of the laser cross section acquired in this region. In the experimental results shown in the figure 8 (top), the navigation strategy selects, from $\mathrm{BP} 1$, the branch formed by $\left(\delta_{1}, \theta_{1}\right)$ and $\left(\delta_{3}, \theta_{3}\right)$ to pursue the exploration. Considering the example shown in figure 9 , the window in $\left(\delta_{2}, \theta_{2}\right)$ is discarded and the signals are tracked in the windows associated to $\left(\delta_{1}, \theta_{1}\right)$ and $\left(\delta_{3}, \theta_{3}\right)$.

The plot (figure 8(bottom)) illustrates the evolution of the distances to the objects used as feedback signals in the control loop. There are two distances in the stabilization from $S$ to $V D$ and during the navigation along the branch. When the object $O_{3}$ start being tracked then the control laws are switched and a third signal is considered in the feedback and the robot is stabilized to the corresponding bifurcation point. It is noted that the control laws are stable and robust with regard to the measurement errors as we can observe in the figure.

The figure 10 shows a complete navigation in an indoor structured but unknown environment validating the proposed control methodology. As previously,

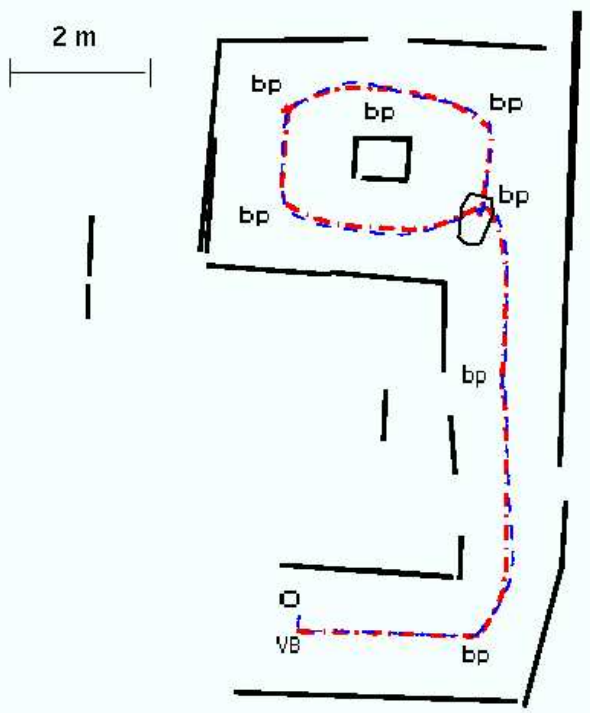

Fig. 10. The application of the control methodology in the robot navigation, localization and map building.

the robot starts from the point $O$, follows the Voronoï branch until it stops at a bifurcation point. Then, it navigates through the successive bifurcation points, marked $b p$, turning in the clockwise sense around the central object. The robot moves without any reference trajectory or off line navigation planning. The trajectories plotted in the figure were calculated and shown 
just for vizualisation purpose.

\section{Conclusions And COMments}

In this paper, we proposed sensor-based navigation functions that enable the robot to safely navigate in an unknown environment, based on the telemetric perception provided by a range finder scanning device mounted on the robot. Feedback control laws were derived from the framework of the task function approach in such a way that the robot is constrained to move on the Voronoï diagram during the exploration of its free workspace. It was shown that this approach does not require any explicit computation of the Voronoï diagram of the environment. A stability analysis of the closed loop control laws with regard to the uncertainties on the perception model was performed and the boundness related to the modeling errors was proved.

The proposed control methodology was experimentally validated in our mobile robot. The stability and robustness of the feedback control laws were verified.

The control methodology presented in this paper is the Part-I of a global navigation methodology we have developed. The construction and the structure of this representation is the subject of another submitted paper (Part-II - Exploration, self-localization and map building). In the Part-II, we revisit the concurrent localization and mapping (CLM) problem. It is proposed there an hybrid modeling methodology of the environment merging in a unique representation metric and topological descriptions. The environment model is structured into several different places, each place having its own metric representation where the robot can be precisely localized. These different places are connected by a global topological graph that shows the accessibility to the BPs of the environment. We will show that embedding the localization and the map building processes in a sensor-based control approach improves the performance in terms of accuracy and robustness. This model will be used for future navigation tasks, where a desired position is given as a goal to the robot. In these cases a trajectory planning step will be required to generate the final trajectory allowing the robot to join a goal which is not located on the Voronoï diagram.

\section{APPENDICES}

\section{The relation between $\hat{e}_{1}$ and $e_{1}$}

A relation between the real $e_{1}$ and the measured $\hat{e}_{1}$ values is derived based on the figure 5 . The physical location of the laser frame on the robot platform is indicated in the figure at the point $(M)$ with coordinates $\left(t x_{R M} t y_{R M}\right)$ on the frame $R$. The frame $M$ is rotated of $\theta_{o f}$ with respect to the frame $R$. The rigid transfor- mation given by the translation $T_{R M}=\left(\begin{array}{ll}t_{x_{R M}} & t_{y_{R M}}\end{array}\right)^{T}$ and the rotation $\theta_{o f}$ is identified experimentally and given by $\hat{T}_{R M}$ and $\hat{\theta}_{o f}$. The quantities $\left|\hat{T}_{R M}-T_{R M}\right|$ and $\left|\hat{\theta}_{o f}-\theta_{o f}\right|$ represent the errors on the parameters of the transformation.

Let us consider $\hat{T}_{C M}$ be the translation between $M$ and $C$ and the orientation of $M$ with respect to $C$ be $\hat{\theta}_{o f}-\beta$, where $C$ as shown in the figure 5 is the frame where the control inputs are calculated. From the figure 5 it follows,

$$
\hat{T}_{C M}=R_{\beta}^{T}\left(\begin{array}{c}
\hat{t}_{x_{R M}}-d \\
\hat{t}_{y_{R M}}
\end{array}\right)=\left(\begin{array}{c}
\hat{t}_{x} \\
\hat{t}_{y}
\end{array}\right)
$$

where,

$$
R_{\beta}=\left(\begin{array}{cc}
\cos (\beta) & -\sin (\beta) \\
\sin (\beta) & \cos (\beta)
\end{array}\right)
$$

The measurements $\left(\theta^{\prime}, \delta^{\prime}\right)$ in the figure 5 , called here $\left(\theta_{i}^{\prime}, \delta_{i}^{\prime}\right)$, are projected into the frame $C$ considering perfect parameters in the transformation $M \rightarrow C$ by,

$$
\left\{\begin{aligned}
\delta_{i}-\delta_{i}^{\prime} & =t_{x} \cos \left(\theta_{i}\right)+t_{y} \sin \left(\theta_{i}\right) \\
\theta_{i}-\theta_{i}^{\prime} & =\theta_{o f}-\beta
\end{aligned}\right.
$$

and using the measured parameters $\hat{T}_{C M}$ and $\hat{\theta}_{o f}$,

$$
\left\{\begin{aligned}
\hat{\delta}_{i}-\delta_{i}^{\prime} & =\hat{t}_{x} \cos \left(\hat{\theta}_{i}\right)+\hat{t}_{y} \sin \left(\hat{\theta}_{i}\right) \\
\hat{\theta}_{i}-\theta_{i}^{\prime} & =\hat{\theta}_{o f}-\beta
\end{aligned}\right.
$$

Some relations used in the development below are obtained from equations (A.2) and (A.3) with $i, j \in$ $\{1,2\}$ :

$$
\begin{aligned}
& \frac{\theta_{i}-\theta_{j}}{2}=\frac{\hat{\theta}_{i}-\hat{\theta}_{j}}{2} \\
& \frac{\theta_{i}+\theta_{j}}{2}-\frac{\hat{\theta}_{i}+\hat{\theta}_{j}}{2}=\theta_{o f}-\hat{\theta}_{o f}
\end{aligned}
$$

Let us consider additive bounded errors $\left\{\varepsilon_{x}, \varepsilon_{y}, \varepsilon_{o f}\right\}$ in the measured parameters so that,

$$
\begin{aligned}
\hat{T}_{C M} & =\left(\begin{array}{c}
\hat{t}_{x}+\varepsilon_{x} \\
\hat{t}_{y}+\varepsilon_{y}
\end{array}\right) \\
\hat{\theta}_{o f} & =\theta_{o f}+\varepsilon_{o f}
\end{aligned}
$$

Replacing (A.5) and (A.6) in (A.3) and taking $\theta_{i}$ as in (A.2) yields:

$$
\hat{\theta}_{i}=\theta_{i}+\varepsilon_{o f}
$$

and

$$
\begin{gathered}
\hat{\delta}_{i}-\delta_{i}^{\prime}=\left(t_{x}+\varepsilon_{x}\right) \cos \left(\theta_{i}+\varepsilon_{o f}\right)+ \\
+\left(t_{y}+\varepsilon_{y}\right) \sin \left(\theta_{i}+\varepsilon_{o f}\right)
\end{gathered}
$$

Expanding (A.8) and considering the following trigonometric approximations,

$$
\varepsilon_{o f} \rightarrow 0 \Rightarrow\left\{\begin{array}{l}
\cos \left(\theta_{i}+\varepsilon_{o f}\right)=\cos \left(\theta_{i}\right)-\varepsilon_{o f} \sin \left(\theta_{i}\right) \\
\sin \left(\theta_{i}+\varepsilon_{o f}\right)=\sin \left(\theta_{i}\right)+\varepsilon_{o f} \cos \left(\theta_{i}\right)
\end{array}\right.
$$


(A.8) can be rewritten as,

$$
\begin{aligned}
& \hat{\delta}_{i}-\delta_{i}^{\prime}=t_{x} \cos \left(\theta_{i}\right)+t_{y} \sin \left(\theta_{i}\right)+ \\
& \quad+\left[\begin{array}{ll}
\hat{t}_{x} \varepsilon_{o f}-\varepsilon_{y} & \hat{t}_{y} \varepsilon_{o f}+\varepsilon_{x}
\end{array}\right]\left(\begin{array}{c}
-\sin \left(\theta_{i}\right) \\
\cos \left(\theta_{i}\right)
\end{array}\right)
\end{aligned}
$$

and with (A.2) it results,

$$
\hat{\delta}_{i}=\delta_{i}+\left[\hat{t}_{x} \varepsilon_{o f}-\varepsilon_{y} \hat{t}_{y} \varepsilon_{o f}+\varepsilon_{x}\right]\left(\begin{array}{c}
-\sin \left(\theta_{i}\right) \\
\cos \left(\theta_{i}\right)
\end{array}\right)
$$

Finally a relation between the tasks $\hat{e}_{1}$ and $e_{1}$ is obtained considering the expressions of the navigation task $e_{1}$ defined in (10) and with the relations (A.7) and (A.9) as it follows.

The task function $e_{1}$, in (10), defined in the controlled frame $C$ is written as:

$$
e_{1}=\left(\begin{array}{c}
\delta_{1}-\delta_{2} \\
\frac{\theta_{1}+\theta_{2}}{2}
\end{array}\right)=\left(\begin{array}{c}
e_{1}(1) \\
e_{1}(2)
\end{array}\right)
$$

and the measured task $\hat{e}_{1}$ is given as,

$$
\hat{e}_{1}=\left(\begin{array}{c}
\hat{\delta}_{1}-\hat{\delta}_{2} \\
\frac{\hat{\theta}_{1}+\hat{\theta}_{2}}{2}
\end{array}\right)=\left(\begin{array}{c}
\hat{e}_{1}(1) \\
\hat{e}_{1}(2)
\end{array}\right)
$$

Taking (A.7) and (A.9) in (A.11),

$$
\hat{e}_{1}=e_{1}+\hat{F}\left(\hat{t}_{x}, \hat{t}_{y}, \hat{\theta}_{1}, \hat{\theta}_{2}\right)
$$

where the vector $F$ is defined as,

$$
F=\left[\begin{array}{c}
-2 \sin \left(\frac{\theta_{1}-\theta_{2}}{2}\right) \hat{T}_{\varepsilon}\left(\begin{array}{c}
\cos \left(\frac{\theta_{1}+\theta_{2}}{2}\right) \\
\sin \left(\frac{\theta_{1}+\theta_{2}}{2}\right)
\end{array}\right) \\
\varepsilon_{o f}
\end{array}\right]
$$

with the relations (A.4) it comes,

$$
\hat{F}=\left[-2 \sin \left(\frac{\hat{\theta}_{1}-\hat{\theta}_{2}}{2}\right) \hat{T}_{\varepsilon}\left(\begin{array}{c}
\cos \left(\frac{\hat{\theta}_{1}+\hat{\theta}_{2}+\varepsilon_{o f}}{\hat{\theta}_{1} \hat{\theta}_{2}}\right) \\
\sin \left(\frac{\varepsilon_{o f}}{2}\right)
\end{array}\right)\right](\mathrm{A}
$$

and the vector $\hat{T}_{\varepsilon}$ as,

$$
\hat{T}_{\varepsilon}=\left[\begin{array}{ll}
\hat{t}_{x} \varepsilon_{o f}-\varepsilon_{y} & \hat{t}_{y} \varepsilon_{o f}+\varepsilon_{x}
\end{array}\right]
$$

The vector $F$ represents the configuration of the environment and it depends on the measured variables, so that from (A.12) it results,

$$
\left\{\varepsilon_{x}, \varepsilon_{y}, \varepsilon_{o f}\right\} \rightarrow\{0\} \quad \Rightarrow \quad \hat{e}_{1}=e_{1}
$$

and,

$$
\dot{\hat{e}}_{1}=\dot{e}_{1}
$$

It is shown in the following that the additive term $F$ in (A.12) represents a bias in the regulation of the real task $e_{1}$, so that when the measured task $\hat{e}_{1}$ is regulated to zero in the task configuration space then, the real task $e_{1}$ is stabilized at a point $e_{1}^{\star}$ into a region around the origin.

Let us note from (A.11) that when the measured task $\hat{e}_{1}$ is regulated to zero then,

$$
\hat{e}_{1} \rightarrow 0 \Rightarrow\left\{\begin{array}{l}
\hat{\theta}_{1}=-\hat{\theta}_{2} \\
\frac{\hat{\theta}_{1}-\hat{\theta}_{2}}{2}=\hat{\theta}
\end{array}\right.
$$

and with relations (A.4),

$$
\begin{aligned}
& \frac{\theta_{1}+\theta_{2}}{2}=-\varepsilon_{o f} \\
& \frac{\theta_{1}-\theta_{2}}{2}=\hat{\theta}
\end{aligned}
$$

Replacing (A.14) in the expression of $\hat{F}$ in (A.12) it follows that when $\hat{e}_{1} \rightarrow 0$ so,

$$
\begin{aligned}
0 & =e_{1}^{\star}+\left[\begin{array}{c}
-2 \sin (\hat{\theta}) \hat{T}_{\varepsilon}\left(\begin{array}{l}
1 \\
0
\end{array}\right) \\
\varepsilon_{o f}
\end{array}\right] \\
e_{1}^{\star} & =\left(\begin{array}{l}
2 \sin (\hat{\theta})\left(\hat{t}_{x} \varepsilon_{o f}-\varepsilon_{y}\right) \\
-\varepsilon_{o f}
\end{array}\right) \\
e_{1}^{\star} & =\operatorname{bias}\left(\hat{t}_{x}, \hat{t}_{y}, \varepsilon_{o f}\right)
\end{aligned}
$$

Then it is noted from (A.15) that the stabilization point $e_{1}^{\star}$ is a function of the parameters errors $\left\{\varepsilon_{x}, \varepsilon_{y}, \varepsilon_{o f}\right\}$ of the rigid transformation between the sensor frame $M$ and the controlled frame $C$. The origin of the controlled frame is stabilized to a region $\operatorname{bias}\left(\hat{t}_{x}, \hat{t}_{y}, \varepsilon_{o f}\right)$ around the Voronoï branch of the environment.

The measurements performed in the laser frame $\left(\theta_{i}^{\prime}, \delta_{i}^{\prime}\right)$ are considered perfect. It is shown in section $\mathrm{V}$, Theorems 1 and 2, that the control systems are stable with a large bound on the errors of the transformation $T_{R M}$, then the noise on the laser measurements does not affect the stability of the control systems.

\section{The proof of the Theorem 2}

The matrix $A_{3}$ in the system (63) is written as:

$$
\begin{aligned}
& A_{3}=\left[\begin{array}{c}
\frac{\sin \left(\frac{\theta_{1}-\theta_{2}}{2}\right) \sin \left(\frac{\theta_{1}+\theta_{2}}{2}-\frac{\hat{\theta}_{1}+\hat{\theta}_{3}}{2}\right)}{\sin \left(\frac{\hat{\theta}_{1}-\hat{\theta}_{2}}{2}\right) \sin \left(\frac{\hat{\theta}_{2}-\hat{\theta}_{3}}{2}\right)} \\
\left.\frac{\sin \left(\frac{\theta_{1}-\theta_{3}}{2}\right) \sin \left(\frac{\theta_{1}+\theta_{3}}{2}-\hat{\theta}_{1}+\hat{\theta}_{3}\right.}{2}\right) \\
\sin \left(\frac{\hat{\theta}_{1}-\hat{\theta}_{2}}{2}\right) \sin \left(\frac{\hat{\theta}_{2}-\hat{\theta}_{3}}{2}\right) \\
0
\end{array}\right. \\
& \left.\begin{array}{cc}
-\frac{\sin \left(\frac{\theta_{1}-\theta_{2}}{2}\right) \sin \left(\frac{\theta_{1}+\theta_{2}}{2}-\frac{\hat{\theta}_{1}+\hat{\theta}_{2}}{2}\right)}{\sin \left(\frac{\hat{\theta}_{1}-\hat{\theta}_{3}}{2}\right) \sin \left(\frac{\hat{\theta}_{2}-\hat{\theta}_{3}}{2}\right)} & 0 \\
-\frac{\sin \left(\frac{\theta_{1}-\theta_{3}}{2}\right) \sin \left(\frac{\theta_{1}+\theta_{3}}{2}-\frac{\hat{\theta}_{1}+\hat{\theta}_{2}}{2}\right)}{\sin \left(\frac{\hat{\theta}_{1}-\hat{\theta}_{3}}{2}\right) \sin \left(\frac{\hat{\theta}_{2}-\hat{\theta}_{3}}{2}\right)} & 0 \\
0 & 1
\end{array}\right]
\end{aligned}
$$

It is well known that the convergence of the system (63) to the unique equilibrium solution $\hat{e}_{3}=0$ is global 
asymptotic if $A_{3}$ is positive definite, $A_{3}>0\left(A_{3_{(3 \times 3)}}>\right.$ 0 in the sense that $\left.x^{T} A_{3} x>0 \forall x_{(3 \times 1)} \neq 0\right)$, if all its eigenvalues are positive (or have positive complex real part).

Defining,

$$
\begin{aligned}
M & =\sin \left(\frac{\theta_{1}+\theta_{3}}{2}-\frac{\hat{\theta}_{1}+\hat{\theta}_{2}}{2}\right) \\
N & =\sin \left(\frac{\theta_{1}+\theta_{2}}{2}-\frac{\hat{\theta}_{1}+\hat{\theta}_{3}}{2}\right) \\
e_{o f} & =\hat{\theta}_{o f}-\theta_{o f}
\end{aligned}
$$

the eigenvalues of $A_{3}$ are done as:

$$
\begin{aligned}
\zeta_{1}= & 1 \\
\zeta_{2}= & -\frac{1}{2 \sin \left(\frac{\hat{\theta}_{2}-\hat{\theta}_{3}}{2}\right)}(M-N \\
& \left.+\sqrt{(M+N)^{2}-4 \sin ^{2}\left(e_{o f}\right)}\right) \\
\zeta_{3}= & \frac{1}{2 \sin \left(\frac{\hat{\theta}_{2}-\hat{\theta}_{3}}{2}\right)}(-M+N \\
& \left.+\sqrt{(M+N)^{2}-4 \sin ^{2}\left(e_{o f}\right)}\right)
\end{aligned}
$$

Considering the geometry in the figure 5 the following relations can be written, with $l \in\{1,2,3\}$ :

$$
\begin{aligned}
& \theta_{l}=\theta_{l}^{\prime}+\theta_{o f}-\beta \\
& \hat{\theta}_{l}=\theta_{l}^{\prime}+\hat{\theta}_{o f}-\beta
\end{aligned}
$$

and $\forall l, k \in\{1,2,3\}$,

$$
\begin{aligned}
e_{o f} & =\frac{\hat{\theta}_{l}+\hat{\theta}_{k}}{2}-\frac{\theta_{l}-\theta_{k}}{2} \\
\frac{\theta_{1}+\theta_{3}}{2}-\frac{\hat{\theta}_{1}+\hat{\theta}_{2}}{2} & =-e_{o f}+\frac{\hat{\theta}_{1}+\hat{\theta}_{3}}{2}-\frac{\hat{\theta}_{1}+\hat{\theta}_{2}}{2} \\
& =-e_{o f}-\frac{\hat{\theta}_{2}-\hat{\theta}_{3}}{2} \\
\frac{\theta_{1}+\theta_{2}}{2}-\frac{\hat{\theta}_{1}+\hat{\theta}_{3}}{2} & =-e_{o f}+\frac{\hat{\theta}_{1}+\hat{\theta}_{2}}{2}-\frac{\hat{\theta}_{1}+\hat{\theta}_{3}}{2} \\
& =-e_{o f}-\frac{\hat{\theta}_{2}-\hat{\theta}_{3}}{2}
\end{aligned}
$$

then with equations (B.4) and (B.2),

$$
\begin{aligned}
& M-N=-2 \sin \left(\frac{\hat{\theta}_{2}-\hat{\theta}_{3}}{2}\right) \cos \left(e_{o f}\right) \\
& M+N=-2 \sin \left(e_{o f}\right) \cos \left(\frac{\hat{\theta}_{2}-\hat{\theta}_{3}}{2}\right) \\
& \sqrt{(M+N)^{2}-4 \sin ^{2}\left(e_{o f}\right)}=2 \sin \left(e_{o f}\right) \sin \left(\frac{\hat{\theta}_{2}-\hat{\theta}_{3}}{2}\right) i
\end{aligned}
$$

where $i=\sqrt{-1}$.

The real part of the complex $\zeta_{2}$ and $\zeta_{3}$ are written below taking (B.5) in (B.3),

$$
\begin{aligned}
R\left(\zeta_{2}\right) & =-\frac{1}{2 \sin \left(\frac{\hat{\theta}_{2}-\hat{\theta}_{3}}{2}\right)}\left(-2 \sin \left(\frac{\hat{\theta}_{2}-\hat{\theta}_{3}}{2}\right) \cos \left(e_{o f}\right)\right) \\
& =\cos \left(e_{o f}\right)=\cos \left(\hat{\theta}_{o f}-\theta_{o f}\right) \\
R\left(\zeta_{3}\right) & =\frac{1}{2 \sin \left(\frac{\hat{\theta}_{2}-\hat{\theta}_{3}}{2}\right)} 2 \sin \left(\frac{\hat{\theta}_{2}-\hat{\theta}_{3}}{2}\right) \cos \left(e_{o f}\right) \\
& =\cos \left(e_{o f}\right)=\cos \left(\hat{\theta}_{o f}-\theta_{o f}\right)
\end{aligned}
$$

Then $R\left(\zeta_{2}\right)>0$ and $R\left(\zeta_{3}\right)>0$ if, and only if,

$$
\left|\hat{\theta}_{o f}-\theta_{o f}\right| \neq \frac{\pi}{2}
$$

\section{REFERENCES}

[1] R.C.Arkin. "Navigational path planning for a vision-based mobile robot", Robotica, vol.7, pp. 49-63, Jan. 1989.

[2] H.Choset, K.Nagatani. "Topological simultaneous localization and mapping (SLAM): toward exact localization without explicit localization", IEEE Transactions on Robotics and Automation, vol.17, no. 2, p. 125-137, April 2001.

[3] R.T.M'Closkey, R.M.Murray. "Exponential stabilization of driftless nonlinear control systems using homogeneous feedback", IEEE Transactions on Automatic Control, vol.42, no.5, pp. 614-628, May 1997.

[4] Gaspar, J., Winters, N., Santos-Victor, J. "Vision-based navigation and environmental representations with an omnidirectional camera", IEEE Transactions on robotics and automation, vol. 16, no. 6, pp. 890-898, 2000.

[5] B.Espiau, F.Chaumette, P.Rives. "A New Approach to Visual Servoing in Robotics", IEEE Trans. Robotics and Automation, v. 8, no. 3, pp. 313-326, June 1992.

[6] T.Hamel, D.Meizel. "On robustness and precision of mobile robot missions", Automatica, vol.37, no.3, pp.437-444, March 2001.

[7] M.Khatib, B.Bouilly, T.Simeon, R.Chatila."Indoor navigation with uncertainty using sensor-based motions",Proc. IEEE International Conference on Robotics and Automation, vol. 4, pp. 3379-3384, April 1997.

[8] L.E.Kavraki, M.N.Kolountzakis, J.-C.Latombe, "Analysis of Probabilistic Road-maps for Path Planning", IEEE Trans. on Rob. and Automation, v.14, n.1, pp.166-171, Feb. 1998.

[9] Kuipers, B., Byun, Y.T., "A robot exploration and mapping strategy based on semantic hierarchy of spatial representations", Robotics and autonomous systems, vol. 8, no. 1-2, pp. 47-63, 1991.

[10] J.P.Laumond, T.Simeon, R.Chatila, G.Giralt." Trajectory planning and motion control for mobile robots", Geometry and Robotics Workshop Proceedings, pp.133-149, Toulouse, France, 1988

[11] J.C.Latombe. "Robot motion planning", Kluwer, 651p., 1991.

[12] D.Miller." A spatial representation system for mobile robots",Proc. IEEE Int. Conf. on Robotics and Automation, pp. 122-127, St. Louis, MO, USA, March 1985.

[13] P.Morin, C.Samson. "Practical stabilization of a class of nonlinear systems. Application to chain systems and mobile robots", Proc 39th IEEE Conference on Decision and Control, vol.3, pp. 2989-2994, Australia, Dec. 2000.

[14] K.Nagatani, H.Choset, "Toward robust Sensor Based Exploration by Constructing Reduced Generalized Voronoï Graph", Proc. IEEE/RSJ Int. Conf. on Intelligent Robots and Systems(IROS'99), v.3, pp. 1687-1692, 1999.

[15] N.S.V.Rao."Robot navigation in unknown generalized polygonal terrains using vision sensors", IEEE Transactions on Systems, Man and Cybernetics, vol.25, no. 6, p. 947-962, June 1995.

[16] P. Rives and R. Pissard-Gibollet and L. Pelletier. "Sensorbased Tasks: From the Specification to the Control Aspects", 6th Int. Symposium on Robotics and Manufacturing, Montpellier, France, May 1996.

[17] A.Saffiotti, K.Konolige, E.H.Ruspini. "A multivalued logic approach to integrating planning and control", Artificial Intelligence, vol.76, no.1-2, pp.481-526, July 1995.

[18] C.Samson, B.Espiau, M.Le Borgne. "Robot Control: The Task Function Approach", Oxford, England: Oxford University Press, 1991.

[19] C.Samson. "Path following and time-varying feedback stabilization of a wheeled mobile robot",Int. Conf. on Automation, Robotics and Computer Vision (ICARCV'g2), Singapore, Sept. 1992

[20] O.J.Sordalem, C.Canudas. "Path following and stabilization of a mobile robot", Proc. IEEE International Conference on Robotics and Automation, vol.3, pp.2158-2163, 1993.

[21] R.Swain Oropeza, M.Devy, S.Hutchinson. "Sensor based navigation in cluttered environments", Proc. IEEE/RSJ Int. 
Conf. on Intelligent Robots and Systems(IROS), pp.16621669, Maui, HI, USA, 2001.

[22] A.Tayebi, M.Tadjine, A.Rachid. "Invariant manifold approach for the stabilization of nonholonomic chained systems: application to a mobile robot", Nonlinear Dynamics, vol.24, no.2, pp. 167-181, Feb. 2001.

[23] Wenjie Dong, Yangsheng Xu, Wei Huo. "On stabilization of uncertain dynamic nonholonomic systems", International Journal of Control, vol.73, no.4, pp. 349-359, March 2000. 